\title{
Enhanced electrical properties of ferroelectric thin films by ultraviolet radiation
}

\author{
Neil McN. Alford and Peter Kr. Petrov ${ }^{\text {a) }}$ \\ Center for Physical Electronics and Materials, Faculty of Engineering, Science and the Build Environment, \\ London South Bank University, 103 Borough Road, London SE1 OAA, United Kingdom \\ Aleksandr G. Gagarin, Andrey B. Kozyrev, Aleksandr I. Sokolov, \\ Oleg I. Soldatenkov, and Valery A. Volpyas \\ Saint Petersburg Electrotechnical University, 5 Professor Popov Street, St. Petersburg, 197376, Russia
}

(Received 23 March 2005; accepted 4 October 2005; published online 22 November 2005)

\begin{abstract}
Ferroelectric films in the paraelectric phase exhibit two undesirable properties: hysteresis in the voltage-capacitance characteristics and a significant relaxation time of the capacitance. Our experiments show that suppression of both of these is achieved by using UV radiation with wavelengths corresponding to the material forbidden gap. Experimentally we also observed UV radiation induced modulation of thin film permittivity without an applied electric field. The observed phenomena are believed to have the same origin: UV light generates nonequilibrium charge carriers that screen out local electric field induced by defects and interfaces inside ferroelectric thin films and change films effective dielectric properties. (c) 2005 American Institute of Physics.
\end{abstract}

[DOI: $10.1063 / 1.2137466]$

Ferroelectric materials have been the subject of extensive research for over 50 years. However, recent investigations of thin films of $\mathrm{SrTiO}_{3}, \mathrm{BaTiO}_{3}$, and their solid solution $\mathrm{Ba}_{x} \mathrm{Sr}_{1-x} \mathrm{TiO}_{3}$ (BSTO), motivated by the prospect of new applications such as electrically controllable microwave devices ${ }^{1,2}$ have uncovered many complexities not previously recognized. The most effort has been aimed at the optimization of thin film fabrication processes, i.e., to the microwave loss reduction (microwave loss tangent of ferroelectric thin films is much higher than that of corresponding bulk crystals), ${ }^{3,4}$ increasing the tunability, ${ }^{5}$ and improving the temperature stability. ${ }^{6,7}$ These points are now better understood, but the issue of the residual polarization and the hysteresis phenomenon observed under varying bias voltage, resulting in slow relaxation of dielectric constant of ferroelectric films in paraelectric phase (above the Curie temperature), ${ }^{8}$ has not been properly addressed.

In principle, ferroelectric materials in the paraelectric phase should not exhibit hysteresis in the $C(V)$ behavior (voltage-capacitance characteristics). ${ }^{9}$ They should demonstrate also very short response time comparable with the period of the lattice soft mode oscillations and the microwave $4 \mathrm{GHz}$ intermodulation distortion measurements (subnanosecond scale of response time) ${ }^{10}$ and nanosecond pulse measurements ${ }^{11}$ appeared to confirm this. However, a more careful analysis of the experimental results ${ }^{11}$ revealed a difference between the variation of dielectric constant under ac and dc voltages, with a dc voltage producing a greater change in dielectric constant. The varactors' fast switching is at the expense of a decreased tunability. Furthermore, the difference between the variation of dielectric constant under ac and dc voltages varied with the magnitude of the applied voltage pulses, their duration and their repetition time making the control of devices problematic. Overcoming this

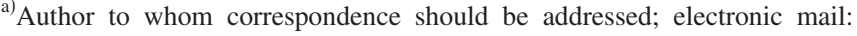
petrovpk@1sbu.ac.uk problem will enable broader application of the ferroelectrics in microwave electronics.

There exist a number of models explaining the residual polarization and dielectric relaxation in ferroelectric thin films. The charge injection ${ }^{12}$ and space charge formation (migration of oxygen vacancies) ${ }^{13}$ models, the Debye-type relaxation model, ${ }^{14}$ and the hopping probabilities distribution model $^{15}$ are among them. However, the real physical mechanisms of the discussed phenomena require further clarification.

In this letter we present a method, validated by experimental results, to solve the hysteresis problem in ferroelectric thin films using UV light radiation.

Previous work examining the application of UV radiation to ferroelectrics considered mainly bulk ferroelectric materials and were devoted to the photocontrollable phase transitions and the UV light induced shift of the Curie temperature. ${ }^{16-18}$ They were motivated by the fact that the contribution from the electronic subsystem (excited atom states, nonequilibrium charge carriers, etc.) to the free energy of the crystal might be substantial. The photoferroelectric effects in the neutral ferroelectric core (with no internal electrical field) are due to the optical excitation of the nonequilibrium charge carriers. ${ }^{19}$

In this investigation, we irradiated thin BSTO films in the paraelectric phase by UV light with wavelengths between 350 and $430 \mathrm{~nm}$. UV light with such wave lengths can generate excess charge carriers in the films since the corresponding photon energies $(2.9-3.7 \mathrm{eV})$ are close to the forbidden gap of BSTO material $(3.3-3.8 \mathrm{eV}) .{ }^{20}$ In turn, the excess charge carriers can affect the electrical properties of the films by suppressing the nonuniform charge distribution and screening charged defects.

The structures investigated were planar capacitors formed on $\mathrm{Ba}_{0.3} \mathrm{Sr}_{0.7} \mathrm{TiO}_{3}$ films deposited by rf magnetron sputtering and laser ablation on $\mathrm{Al}_{2} \mathrm{O}_{3}$ and $\mathrm{LaAlO}_{3}$ substrates, respectively. Details concerning the deposition processes can be found elsewhere. ${ }^{21,22}$ Various samples with 


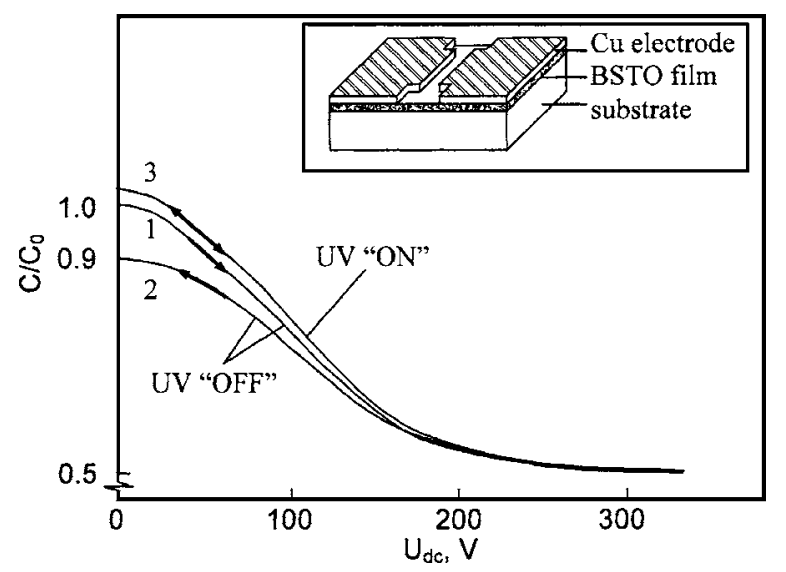

FIG. 1. Capacitance of the BSTO capacitor vs dc bias voltage at absence of ultraviolet exposure (curves 1 and 2 refer to the increase and decrease of bias voltage, respectively) and at ultraviolet exposure (curve 3). Insert shows the structure of ferroelectric film planar capacitor investigated. $C_{0}$ $=1.2 \mathrm{pF}$.

thickness between 0.5 and $1 \mu \mathrm{m}$ were investigated. The $\mathrm{Cu}-$ rie temperature of all samples was measured to be about $140 \mathrm{~K}$, which is in a good agreement with that measured for the bulk materials. The estimated dielectric permittivity $\left(\varepsilon^{\prime}\right)$ at zero dc voltage and room temperature varied between 300 and 500. The electrodes (bilayers of $\mathrm{Au} / \mathrm{Ti}$ or $\mathrm{Cu} / \mathrm{Cr}$ ) were formed by thermal evaporation followed by lift-off or wet etching, respectively. The metal layer thickness was between 0.5 and $1 \mu \mathrm{m}$, while the gap between the electrodes varied from 5 to $20 \mu \mathrm{m}$.

The sample capacitance was measured at room temperature $(300 \mathrm{~K})$ under reverse dc bias in $0-300 \mathrm{~V}$ range, using LRC meters E7-12 (1 MHz) and Agilent 4287A (1 GHz). The error in both measurements did not exceed few femtoFarads. The results obtained were similar for all samples at rf and microwave frequencies, therefore experimental data presented below are in arbitrary units and measurement frequency is not mentioned.

Three types of experiment were performed.

(1) Voltage-capacitance characteristic of the capacitors were measured with and without UV irradiation. The UV light source was a GaAs light emitting diode with wavelength $370 \mathrm{~nm}$ and power density $7.5 \mathrm{~mW} / \mathrm{cm}^{2}$. Typical results are presented in Fig. 1. Curves 1 and 2 show the reversible change of the sample capacitance under dc bias changed from 0 to $300 \mathrm{~V}$ and back to $0 \mathrm{~V}$ without $\mathrm{UV}$ irradiation. As one can see, a well-pronounced hysteresis of the capacitance takes place. Under UV light, a slight increase of the capacitance is observed at $0 \mathrm{~V}$ (see curve 3), which results in steeper behavior of the voltage-capacitance characteristics at low dc bias. At high dc bias (close to the $U_{\max }$ ), however, the characteristics become identical. On decreasing voltage back to zero, the measured capacitance values are identical with those previously measured on the voltage increase, i.e., no hysteresis is observed. Similar results were obtained for all measured samples irradiated with UV light with wavelength between 350 and $430 \mathrm{~nm}$. At longer wavelengths, however, the removal of hysteresis was not complete: it was reduced substantially in comparison with the case without UV irradiation, but was still detectable.

A possible explanation of the described phenomenon (hysteresis disappearance) is that the UV photons with energy close to the BSTO band gap $(3.3-3.8 \mathrm{eV})$ generate nonDownloaded 28 Nov 2005 to 129.16.43.88. Redistribution subject

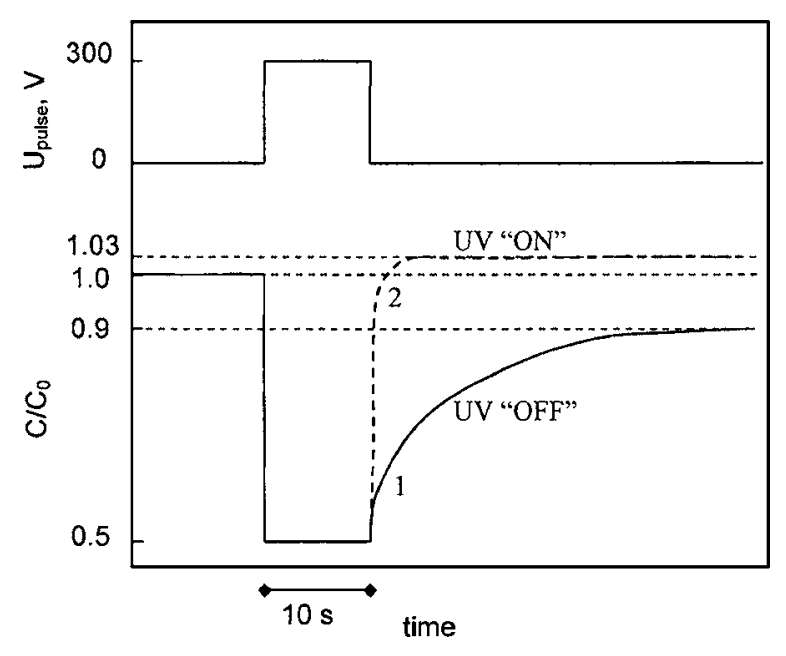

FIG. 2. Evolution in time of the capacitance of the BSTO capacitor after the pulsed control voltage is switched off without (curve 1) and under (curve 2) the exposure of ultraviolet light. $C_{0}=1.2 \mathrm{pF}$.

equilibrium charge carriers, which neutralize the space charge trapped in the film itself and/or at the film/substrate, film/electrode interface. The nature and origin of these defects are the subject of continued investigation. The incomplete suppression of the hysteresis in some of the experiments with longer UV wavelength is believed to be due to the insufficient energy of the UV photons. It should be noted that the capacitor structures were not optimised for these measurements.

(2) Measurements were made to investigate the influence of the UV light irradiation on the capacitance/permittivity relaxation time after the end of control voltage pulse. Rectangular pulses with amplitudes up to $300 \mathrm{~V}$ and duration between 10 and $30 \mathrm{~s}$ were applied to the same capacitor structures. The capacitance was measured with and without UV light. Typical results are presented in Fig. 2. Curves 1 and 2 represent the capacitor response to a rectangular pulse with $10 \mathrm{~s}$ duration showing conventional relaxation type behavior. Without UV light, the capacitance/permittivity relaxation achieved $90 \%$ of the initial value after several tens of seconds (curve 1, Fig. 2). With UV irradiation the relaxation time was significantly reduced (curve 2, Fig. 2).

(3) In both experiments, all samples under UV irradiation and $0 \mathrm{~V}$ bias exhibited capacitance higher than that measured in the virgin state. This led us to the idea of the third experiment: modulation of the BSTO capacitance by an irradiation of modulated ultraviolet light. The results are presented in Fig. 3. As one can see, the change in capacitance follows exactly the time modulated $(0.5 \mathrm{~Hz}) \mathrm{UV}$ radiation. Again, although the experimental setup used was unable to measure the exact value of the relaxation time, it was well below a few milliseconds (the equipment refresh time). We believe that the origin of the observed phenomena is the photoinduced generation of nonequilibrium charge carriers which reduces a local electric field in the film itself and/or at the film/substrate, film/electrode interface. This affects the local dielectric permittivity and changes the effective permittivity of the sample. The change in absolute capacitance value was about $3 \%$. However, as mentioned earlier, the capacitor design was not optimized for these measurements.

In principle, the variation of sample capacitance/ permittivity under UV radiation may be also due to its heating. To exclude this possibility, we estimated the upper 


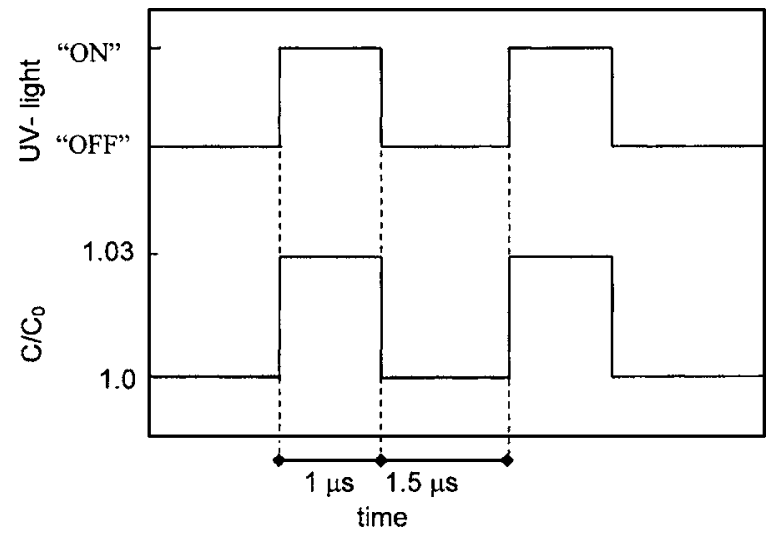

FIG. 3. The modulation of the BSTO capacitance due to modulated ultraviolet light. No bias voltage was applied. $C_{0}=1.2 \mathrm{pF}$.

bound of UV radiation induced variation of the sample temperature. Assuming that all the UV light power is absorbed by thin film, the UV induced change in sample temperature was estimated to be less than $0.01 \mathrm{~K}$, which is well below the value that could produce the measured change in sample capacitance.

In conclusion, our investigation shows that by using UV radiation with wavelengths corresponding to the BSTO thin film forbidden gap, it is possible to remove or significantly suppress the hysteresis in the voltage-capacitance characteristics of BSTO based nonlinear capacitors. We also observe a reduction in the capacitance/permittivity relaxation time. Experimentally we demonstrated that BSTO thin film permittivity is sensitive to the UV radiation even without an applied electric field. The observed phenomena are considered to have the same origin. Namely, UV light causes generation of nonequilibrium charge carriers that screen out local electric field induced by defects and interfaces inside the BSTO thin films and alter their effective dielectric properties.

This work was partly supported by the Engineering and Physical Sciences Research Council (EPSRC), UK. A.I.S. acknowledges the financial support of the Russian Foundation for Basic Research under Grant No. 04-02-16189.
${ }^{1}$ F. W. Van Keuls, R. R. Romanofsky, C. H. Mueller, J. D. Warner, C. L. Canedy, R. Ramesh, and F. A. Miranda, Integr. Ferroelectr. 34, 165 (2001).

${ }^{2}$ A. Kozyrev, A. Ivanov, V. Keis, M. Khasov, V. Osadchy, T. Samoilova, O. Soldatenkov, A. Pavlov, G. Koepf, C. Mueller, D. Galt, and T. Rivkin, IEEE MTT-S Int. Microwave Symp. Dig. 2, 985 (1988).

${ }^{3}$ G. Rupprecht, R. O. Bell, and B. D. Silverman, Phys. Rev. 123, 97 (1961).

${ }^{4}$ A. B. Kozyrev, T. B. Samoilova, A. A. Golovkov, D. A. Kalinikos, E. K. Hollman, V. E. Loginov, A. M. Prudan, D. Galt, C. H. Mueller, T. V. Rivkin, and C. A. Koepf, J. Appl. Phys. 84, 3326 (1998).

${ }^{5}$ S. V. Rasumov, A. V. Tumarkin, M. M. Gaidukov, A. G. Gagarin, A. B. Kozyrev, O. G. Vendik, A. V. Ivanov, O. U. Buslov, V. N. Keys, L. C. Sengupta, and X. Zhang, Appl. Phys. Lett. 81, 1675 (2002).

${ }^{6}$ S. Gevorgyan, P. K. Petrov, Z. G. Ivanov, and E. L. Kollberg, Appl. Phys. Lett. 79, 1861 (2001)

${ }^{7}$ A. Prudan, A. Kozyrev, V. Osadchy, D. Ginley, T. Kaydanova, J. Perkins, J. Alleman, L. Segupta, L. Chiu, and X. Zhang, Integr. Ferroelectr. 58, 1337 (2003).

${ }^{8}$ V. V. Lemanov, B. M. Goltsman, V. K. Yarmarkin, and Yu. A. Boikov, Ferroelectrics 286, 251 (2003).

${ }^{9}$ E. Sawaguchi, A. Kikuchi, and Y. Kodera, J. Phys. Soc. Jpn. 17, 1666 (1962).

${ }^{10}$ A. Kozyrev, A. Ivanov, T. Samoilova, O. Soldatenkov, K. Astafiev, and L. Sengupta, J. Appl. Phys. 88, 5334 (2000).

${ }^{11}$ A. Kozyrev, T. Samoilova, E. Shafranova, T. Kaydanova, D. Ginley, J. Perkins, J. Alleman, L. Segupta, L. Chiu, and X. Zhang, Integr. Ferroelectr. 55, 815 (2003).

${ }^{12}$ I. Stolichnov and A. Tagantsev, J. Appl. Phys. 84, 3216 (1998).

${ }^{13}$ Yu. A. Boikov, B. M. Goltsman, V. K. Yarmarkin, and V. V. Lemanov, Appl. Phys. Lett. 78, 3866 (2001).

${ }^{14}$ M. Schumacher, G. W. Deitz, and R. Waser, Integr. Ferroelectr. 10, 231 (1995).

${ }^{15}$ X. Chen, A. I. Kingon, L. Mantese, O. Auchiello, and K. Y. Hsieh, Integr. Ferroelectr. 3, 355 (1993).

${ }^{16}$ M. Takesada, M. Itoh, T. Yagi, and S.-Y. Koshihara, Ferroelectrics 286, 3 (2003).

${ }^{17}$ V. M. Fridkin, A. A. Grekov, P. V. Ionov, A. I. Rodin, E. A. Savchenko, and K. A. Mikhailina, Ferroelectrics 8, 433 (1974).

${ }^{18}$ S. Ueda, I. Tatsuzaki, and J. Shindo, Phys. Rev. Lett. 18, 453 (1967).

${ }^{19}$ Photoferroelectrics, edited by V. M. Fridkin (Springer, Berlin, 1979).

${ }^{20}$ R. Thielsh, K. Kaemmer, B. Holzapfel, and L. Schultz, Thin Solid Films 301, 203 (1997)

${ }^{21}$ S. V. Razumov and A. V. Tumarkin, Integr. Ferroelectr. 55, 871 (2003).

${ }^{22}$ A. B. Kozyrev, A. V. Ivanov, T. B. Samoilova, O. I. Soldatenkov, L. C. Sengupta, T. V. Rivkin, C. M. Carlson, P. A. Parilla, and D. S. Ginley, Integr. Ferroelectr. 24, 297 (1999). 